\title{
How can research fields be integrated with PET imaging?
}

\author{
Haruhiko Sugimura
}

Published online: 18 July 2012

(c) The International Gastric Cancer Association and The Japanese Gastric Cancer Association 2012

Advances in molecular biology have reasonably driven many pathologists and many of those desiring to become pathologists to investigate the molecular characterization of human tumors. Pathologists specializing in gastric cancer are not exceptions. Microsatellite instability (MSI) in the DNA of human cancer cells has attracted the attention of the research community because MSI sometimes reflects constitutive genetic defects in mismatch repair (MMR) genes. Such information is especially important for the diagnosis of colorectal cancer in patients suspected of having hereditary nonpolyposis colorectal cancer (HNPCC) [1]. While MSI itself is characteristic of tumors, it also reflects genetic traits transmitted from one generation to the next.

MSI was previously assumed to play the same role in gastric cancer as it does in colorectal cancer: that is, many researchers expected to observe frequent MSI in familial gastric cancer cases. In most settings, however, this has not been the case. MSI in gastric cancer is known to occur in response to a deficiency in MMR genes because of promoter methylation. The methylation of $M L H 1$ has been identified in metaplastic gastric mucosa surrounding differentiated adenocarcinoma [2]. Epigenetic changes similar to those occurring in non-tumor gastric mucosa are now being extensively studied in light of their possible use as predictors of the recurrence of gastric cancer after endoscopic submucosal dissection [3]. Of note, the biological and clinical significance of MSI during the advanced stage of gastric cancer has been extensively studied for the past

This editorial refers to the article doi:10.1007/s10120-012-0165-2.

H. Sugimura $(\square)$

Department of Tumor Pathology, Hamamatsu University School

of Medicine, Hamamatsu, Japan

e-mail: hsugimur@hama-med.ac.jp decade. MSI is a molecular marker that is involved in the pathogenesis and progression of gastric cancer either in an independent manner and/or in coordination with pivotal cancer-associated genes, such as $C D H 1$ and MET [4]. Importantly, MSI-positive tumors exhibit changes in the target genes (of the MMR genes) that control critical biological behaviors in tumors [5]. Despite these expectations regarding the use of MSI as a clinically feasible marker, neither clinicians nor pathologists have regarded MSI as a critical factor on which clinical decisions must be based. How do other members of the clinical team view the contribution of MSI? Diagnostic radiologists are usually not familiar with MSI (based on my personal experience), and imaging radiology has been one of the disciplines farthest from research fields examining genetic aberrations in tumors.

In this issue of Gastric Cancer, the work of an interdisciplinary team at the Konkuk University School of Medicine in Seoul, Korea, is reported (Chung et al. [6]); this team has integrated the above-mentioned research fields, combining positron emission tomography (PET) findings with MSI status for the diagnosis of gastric cancer6. Because researchers at this university have continuously reported data on MSI in colorectal and gastric cancer, their data for MSI in gastric cancer is accumulating [7]. Whether Chung et al. [6] intentionally compared PET data and MSI findings or whether their encounter with this correlation was serendipitous is not clear. The key findings of their study were that the presence of fluorodeoxyglucose (FDG) uptake on PET/computed tomography (CT) images $(P=0.001)$ and a higher maximum standardized uptake value (SUVmax) in gastric cancer was linked to the presence of MSI $(P<0.001)$. Many PET researchers have been trying to correlate PET findings with tumor phenotypes [8], but matching such findings to the molecular nature of 
tumors is not an easy achievement in clinical settings, even though many clinicians are aware that MSI can provide useful information regarding tumors that is highly relevant to the present-day management of patients.

One straightforward interpretation of the findings of Chung et al. would be that the apparent association they found between PET/CT detectability and MSI is a secondary effect arising from the tumor size and other factors. Actually, PET/CT detectability is related to the patient's age and the size of the tumor. MSI is also known to be prevalent in advanced gastric cancers among the elderly [9]. Even though the apparent association between PET/CT detectability and MSI could be a secondary coincidence, the reported findings provide several insights as to why this association occurs. FDG-PET/CT detects areas with high glucose intake, which is dependent on the activity of the glucose transporter 1 (GLUT1) protein. The report by Chung et al. [6] in this issue of the journal does not provide GLUT1 (SLC 2A1) expression data, although they mentioned on that; however, several reports have shown GLUT1 overexpression in human tumors. GLUT1 is located at $1 \mathrm{p} 34$, in the vicinity of many genes associated with gastric cancer (including EPHA8, EPHB2, TIE, and $M Y H$ ), and GLUT1 expression in gastric cancer may be related to the phenomenon reported by Chung et al. [6]. This perspective may have broader implications regarding the molecular natures of tumors in terms of personalized therapeutics. GLUT1 is one of the most frequently increased transcripts in colorectal cancer with the $B R A F$ mutation [10], which is a molecular marker highly relevant to personalized therapy for colorectal cancer. Thus, PET positivity and its correlation with MSI may be the first theranostic [11] finding in the field of gastric cancer research and practice. Not many modalities for personalized therapy for gastric cancer, other than human epidermal growth factor receptor 2 (HER2)-Herceptin (trastuzumab), are available at present, but a wider variety of markers, including MSI and associated features, are likely to be available in the near future. For optimists, the information reported here indicating that PET may disclose potentially manipulable characteristics of the gastric cancer genome is encouraging.

\section{References:}

1. Goodenberger M, Lindor NM. Lynch syndrome and MYHassociated polyposis: review and testing strategy. J Clin Gastroenterol. 2011;45:488-500.

2. Guo RJ, Arai H, Kitayama Y, et al. Microsatellite instability of papillary subtype of human gastric adenocarcinoma and hMLH1 promoter hypermethylation in the surrounding mucosa. Pathol Int. 2001;51:240-7.

3. Maekita T, Nakazawa K, Mihara M, et al. High levels of aberrant DNA methylation in Helicobacter pylori-infected gastric mucosae and its possible association with gastric cancer risk. Clin Cancer Res. 2006;12:989-95.

4. Yokozaki H, Yasui W, Tahara E. Genetic and epigenetic changes in stomach cancer. Int Rev Cytol. 2001;204:49-95.

5. Shah SN, Hile SE, Eckert KA. Defective mismatch repair, microsatellite mutation bias, and variability in clinical cancer phenotypes. Cancer Res. 2010;70:431-5.

6. Chung HW, Lee S-Y, Han HS, et al. Gastric cancers with microsatellite instability exhibit high fluorodeoxyglucose uptake in positron emission tomography. Gastric Cancer. 2012.

7. Lee SY, Chung H, Devaraj B, et al. Microsatellite alterations at selected tetranucleotide repeats are associated with morphologies of colorectal neoplasias. Gastroenterology. 2010;139:1519-25.

8. Tsujikawa T, Yoshida Y, Maeda H, et al. Oestrogen-related tumour phenotype: PET characterisation with $18 \mathrm{~F}-\mathrm{FDG}$ and $18 \mathrm{~F}-$ FES. Br J Radiol. 2010.

9. Wang Y, Shinmura K, Guo RJ, et al. Mutational analyses of multiple target genes in histologically heterogeneous gastric cancer with microsatellite instability. Jpn J Cancer Res. 1998;89:1284-91.

10. Yun J, Rago C, Cheong I, et al. Glucose deprivation contributes to the development of KRAS pathway mutations in tumor cells. Science. 2009;325:1555-9.

11. Niu G, Chen X. Molecular imaging with activatable reporter systems. Theranostics. 2012;2:413-23. 